\title{
Creating a molecular atlas of clear cell renal cell
} carcinoma genetics

$\mathrm{C}$ lear cell renal cell carcinoma (ccRCC) represents the most common form of kidney cancer, but the molecular pathogenesis of the disease remains poorly understood. Two recently published studies have sought to remedy this situation by analysing the genetic changes underlying the disease.

In a recent article in Nature, The Cancer Genome Atlas Research Network report their survey of over 400 tumours to identify significantly mutated genes. Using a number of different genomic platforms - including RNA sequencing, DNA methylation arrays, miRNA sequencing, single nucleotide polymorphism (SNP) arrays, reverse phase protein arrays and exome sequencingthe group identified 19 genes that were significantly mutated.

Such mutations commonly involved entire chromosome arms as opposed to focal events, most frequently arm-level events of chromosome $3 p$, the site of the four most commonly mutated genes in ccRCC: VHL, PBRM1, BAP1 and SETD2. Arm-level mutations were also observed on chromosome 14q, which is associated with loss of HIF1A, a gene predicted to drive more aggressive disease.

BAP1 mutation was significantly correlated with poor survival, as were reduced AMP kinase and increased acetylCoA carboxylase, both of which contribute to a metabolic shift towards increased fatty acid synthesis. Overall, a metabolic shift towards a 'Warburg-like effect' was noted, with downregulation of AMPK complex and Krebs cycle genes and upregulation of pentose phosphate pathway genes; fatty acid synthesis genes were associated with poor prognosis.

The Cancer Genome Atlas group suggest the possibility that some of the identified genes could be targeted for future therapeutic options. "These findings, consistent with a Warburg metabolic shift in clear cell kidney cancer with poor survival, suggest that these tumours are dependent on aerobic glycolysis, impaired oxidative phosphorylation and increased fatty acid synthesis," comments corresponding author W. Marston Linehan. "This raises a number of very exciting possibilities about targeting the metabolic basis of clear cell kidney cancer. Hopefully this work will help provide the foundation for the development of effective forms of therapy for patients with this malignancy."

Further to these exciting developments, a second recent paper, published in Nature Genetics, also reports the elucidation of the genetic background of ccRCC using a combination of whole genome/exome sequencing, RNA sequencing and array-based methylation, copy number and expression analyses. Sato et al. used an extended cohort of 204 ccRCC specimens, which were analysed to validate and clarify the effects of major genetic abnormalities.

Similar to the results reported by the Cancer Genome Atlas, Sato and colleagues observed recurrent mutations in targets on chromosome $3 \mathrm{p}$, including $V H L$, PBRM1, BAP1 and SETD2. 94\% of samples exhibited loss of heterozygosity $(\mathrm{LOH})$ at $3 p$, often caused by simple $3 p$ loss. Most samples exhibiting $\mathrm{LOH}$ at $3 \mathrm{p}$ had the second VHL allele affected by somatic mutation or promoter methylation. Furthermore, the team found a novel mutation in the VHL pathway, in TCEB1, which encodes a vital part of the VHL complex. Overall, genetic and epigenetic lesions in the VHL complex accounted for 229 of the 240 specimens, in which $V H L$ and TCEB1 lesions were mutually exclusive, once again emphasizing the importance of VHL complex inactivation in ccRCC.

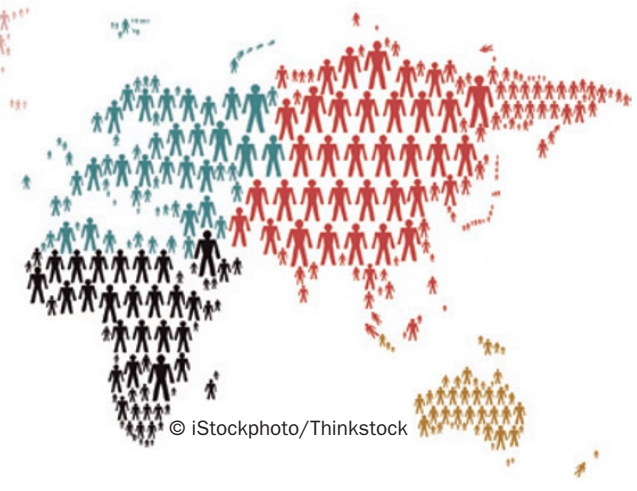

Again echoing the data from the Cancer Genome Atlas, a large number of ccRCC cases involved mutations in the PI3K-AKT-mTOR pathway, as well as in TET2, and KEAP1. TET2 encodes an oxygenase that catalyses a critical step in DNA demethylation, whereas KEAP1 is a component of a complex involved in oxidative stress responses.

The team also classified ccRCC cases into three subgroups based on DNA methylation patterns. Those with a high methylation subtype showed significantly worse prognosis, suggesting that patients with these tumours should be monitored for early detection of metastasis for potential aggressive treatment. The use of integrated molecular analysis in this study has enabled the researchers to piece together a deeper understanding of the interplay of genetic mechanisms affecting ccRCC pathogenesis. "Our study underscores the importance of integrated molecular studies to understand the pathogenesis of human cancers," explains Seishi Ogawa, corresponding author. The team now plan to validate their findings in a larger cohort and to investigate the relationship between mutation status and specific treatment responses.

Annette Fenner

Original articles The Cancer Genome Atlas Research Network. Comprehensive molecular characterization of clear cell renal cell carcinoma. Nature doi:10.1038/nature12222 | Sato, Y. et al. Integrated molecular analysis of clear-cell renal cell carcinoma. Nat. Genet. doi:10.1038/ng.2699 